\title{
Competências e habilidades dos discentes do curso de nutrição para aplicar a avaliação subjetiva global para fins de diagnóstico nutricional
}

\author{
Fábio Costa de Vasconcelos \\ Graduado em Nutrição pela Universidade Federal do Pará. Mestre em Ensino em Saúde na Amazônia pela \\ Universidade do Estado do Pará. Especialista em Nutrição Oncológica pelo Instituto Nacional de Câncer. \\ Especialista em Residência em Nutrição Clínica pela Universidade Federal do Pará. Especialista em \\ Bioestatística pela Universidade Federal do Pará; Coordenador de Pós-Graduação em Nutrição Clínica \\ 凹 biovasconcelos@hotmail.com

\section{Lizomar de Jesus Maués Pereira Moia} \\ Graduada em Medicina pela a Universidade Federal do Pará, Residência em Clínica Médica Fundação Santa \\ Casa de Misericórdia do Pará/Universidade Federal do Pará e Doutora em Fisiologia dos Órgãos e Sistemas - \\ Kagawa University Japão. Professora Dra. Adjunto do Mestrado em Ensino em Saúde na Amazônia \\ Universidade do Estado do Pará \\ \lizomar@ufpa.br
}

\section{Irland Barroncas Gonzaga Martens}

Graduada em Nutrição pela Universidade Federal do Pará, Mestre em Ciências de Alimentos pela Universidade Federal do Amazonas e Doutora em Ciências dos Alimentos pela Universidade de São Paulo. Docente da Universidade Federal do Pará. Atua na área de pesquisa em Nutrição, com ênfase em Nutrição Experimental e análise de minerais por AAS e HG-AAS

\section{Resumo:}

A avaliação subjetiva do estado nutricional de indivíduos enfermos deve ser feita por meio de abordagem multivariada, sendo essencialmente clínica. Assim, a Avaliação Subjetiva Global é vista como um dos melhores métodos para obtenção do diagnóstico do paciente. 0 estudo teve o objetivo de verificar se os discentes concluintes do curso de nutrição apresentam competência e habilidades para aplicar a avaliação subjetiva global para fins de diagnóstico nutricional. A pesquisa foi descritiva, analítica-exploratória, transversal,comabordagem quantitativa. A população foi constituída de cem discentes, de ambos os sexos, regularmente matriculados, concluintes de quatro Instituições de Ensino Superior do curso de Nutrição de B elém/PA. Os dados foramobtidos por meio de questionário semiestruturado, com perguntas fechadas a respeito da percepção dos discentes no processo de avaliação e diagnóstico nutricional pela avaliação subjetiva global. Aplicou-se análise de correspondência para verificar associação das variáveis quantitativas relacionadas com a avaliação subjetiva e as respostas dos discentes das instituições de ensino superior. As variáveis associadas foram: finalidade da ferramenta; classificação do estado nutricional do paciente e técnica pararealizar o exame físico. Destacam-se associações significativas com problemas relacionados à abordagemao paciente, finalidade da avaliação incorreta, realização do exame físico de forma inadequada; dificuldade na interpretação das perguntas de cunho clínico; bem como erros na interpretação dos resultados da avaliação para fins de diagnóstico nutricional do paciente. As dificuldades observadas na aplicação da avaliação subjetiva global limitaram as habilidades e competências adquiridas no âmbito acadêmico pelos discentes.

Palavras-chave: Estudantes, Ensino, Competência Profissional, Avaliação Nutricional, Estado Nutricional. 


\title{
Skills and abilities of nutrition course students to apply global subjective assessment for nutritional diagnosis purposes
}

\begin{abstract}
:
The subjective evaluation of the nutritional status of sick individuals should be done through a multivariate approach, being essentially clinical. Thus, Global Subjective Assessment is seen as one of the best methods for obtaining a patient's diagnosis. The objective of this study was to verify if the final students of the nutrition course present competence and abilities to apply the global subjective evaluation for nutritional diagnosis purposes. The research was descriptive, analytical-exploratory, cross-sectional, with a quantitative approach. The population was constituted of one hundred students, of both sexes, regularly enrolled, graduates of four Institutions of Higher Education of the course of Nutrition of Belém/ PA. The data were obtained through a semi-structured questionnaire, with closed questions about the perception of the students in the evaluation process and nutritional diagnosis by the global subjective evaluation. Correspondence analysis was applied to verify the association of the quantitative variables related to the subjective evaluation and the answers of the students of the higher education institutions. The associated variables were: purpose of the tool; classification of the nutritional state of the patient and technique to perform the physical examination. Significant associations with problems related to the approach to the patient, purpose of the incorrect evaluation, inadequate physical examination are highlighted; difficulty in interpreting clinical questions; as well as errors in the interpretation of the results of the evaluation for purposes of nutritional diagnosis of the patient. The difficulties observed in the application of the global subjective evaluation limited the skills and competences acquired in the academic scope by the students.
\end{abstract}

Keywords: Students, Teaching, Professional Competence, Nutritional Evaluation, Nutritional status.

\section{Competencias y habilidades de los alumnos del curso de nutrición para aplicar la evaluación subjetiva global para fines de diagnóstico nutricional}

\section{Resumen:}

La evaluación subjetiva del estado nutricional de individuos enfermos debe ser hecha por medio de abordaje multivariada, siendo esencialmente clínica. Así,la Evaluación Subjetiva Global es vista como uno de los mejores métodos para obtener el diagnóstico del paciente. El estudio tuvo el objetivo de verificar si los discursos concluyentes del curso de nutrición presentan competencia y habilidades para aplicar la evaluación subjetiva global para fines de diagnóstico nutricional. La investigación fue descriptiva, analítica-exploratoria, transversal, con abordaje cuantitativo. La población fue constituida de cien discentes, de ambos sexos, regularmente matriculados, concluyentes de cuatro Instituciones de Enseñanza Superior del curso de Nutrición de Belém/PA. Los datos fueron obtenidos por medio de un cuestionario semiestructurado, con preguntas cerradas sobre la percepción de los discentes en el proceso de evaluación y diagnóstico nutricional por la evaluación subjetiva global.Se aplicó análisis de correspondencia para verificar la asociación de las variables cuantitativas relacionadas con la evaluación subjetiva y las respuestas de los alumnos de las instituciones de enseñanza superior. Las variables asociadas fueron: propósito de la herramienta; clasificación del estado nutricional del paciente y técnica para realizar el examen físico. Se destacan asociaciones significativas con problemas relacionados al abordaje al paciente, propósito de la evaluación incorrecta, realización del examen físico de forma inade cuada; dificultad en la interpretación de las preguntas de cuño clínico; así como errores en la interpretación de los resultados de la evaluación para fines de diagnóstico nutricional del paciente. Las dificultades observadas en la aplicación de la evaluación subjetiva global limitaron las habilidades y competencias adquiridas en el ámbito académico por los alumnos.

Palabras clave: Estudiantes, Educación, Competencia Profesional, Evaluación nutricional, Estado Nutricional. 


\section{INTRODUÇÃO}

A desnutrição hospitalar é um evento prevalente na atualidade, contribuindo para o aumento do tempo e custo com a hospitalização, piora na qualidade de vida do paciente, incidência elevada de infecções, morbidades e mortalidade, além de redução da função imunológica. Portanto, o estado nutricional é um critério fundamental a ser avaliado no ambiente hospitalar, a fim de manter e/ou recuperar a saúde do paciente (LIM, LIN e DANIELS, 2016; ANDRADE, 2018).

Atualmente, os métodos de avaliação do estado nutricional de pacientes são alvos de vários estudos. O diagnóstico nutricional pode ser determinado por métodos subjetivos, como a avaliação subjetiva global (ASG), permitindo identificar indivíduos desnutridos ou em risco de desnutrição possibilitando intervenção nutricional (RASLAN, 2010; AQUINO e PHILIPPI, 2012; LIM, LIN e DANIELS, 2016).

Dessa forma, a avaliação subjetiva do estado nutricional de indivíduos enfermos, idealmente deve ser feita por meio de abordagem multivariada e, essencialmente clínica. Assim, a Avaliação Subjetiva Global (ASG), padronizada por Detsky et al. (1987), é vista como um dos melhores métodos para obtenção do diagnóstico do paciente. A mesma teve o objetivo de avaliar o estado nutricional de pacientes cirúrgicos, dividindo-os em bem nutridos, desnutrição moderada e desnutrição grave (DIAS, 2011; DOMINGUES, 2018).

Assim, a American Society for Parenteral and Enteral Nutrition - ASPEN (2013), propõe como padrão-ouro o uso da ASG por ser um método simples, de baixo custo, não invasivo e por apresentar boa precisão diagnóstica. Em virtude de boa sensibilidade e especificidade ao predizer infecções pós-operatórias em pacientes cirúrgicos e passou a ser reformulada para ser aplicada em grupos específicos, a fim de aumentar sua reprodutibilidade. Além disso, devido ao seu valor preditivo pode ser aplicada por profissionais de saúde que recebam treinamento prévio (MALONE e HAMILTON, 2013; HANUSCH et al., 2016; PAZ e COUTO, 2016; SOUSA JUNIOR et al., 2016, SANTOS et al., 2017).

A ASG foi desenhada de modo a se obter poucos resultados falso-positivos, isto é, o paciente classificado como desnutrido grave tem poucas chances de ser nutrido ou moderadamente desnutrido. Dessa forma, o questionário o torna um teste de alta especificidade. Além disso, a ASG não é apenas utilizada para classificar o estado nutricional, 
mas também para identificar pacientes que apresentam maior risco de sofrer complicações associadas ao estado nutricional durante sua internação. Logo a ASG é utilizada tanto para o diagnóstico adequado da desnutrição quanto para o prognóstico, fundamental para determinar as intervenções nutricionais (LIM, LIN e DANIELS, 2016; SOUSA JUNIOR et al., 2016; PAZ e COUTO, 2016).

Ressalta-se que a desnutrição, por muitas vezes, não é reconhecida ou não é diagnosticada por profissionais de saúde, entre eles o nutricionista, pois o diagnóstico apropriado requer conhecimento fundamental dos métodos de avaliação. É indicado que as Instituições de Ensino Superior (IES) devam formar nutricionistas capacitados nas diversas áreas de atuação, entre elas, a nutrição clínica, baseada nas competências e habilidades específicas afim de nortear o acadêmico durante o processo de ensino e aprendizagem saúde, conforme as Diretrizes Curriculares Nacionais (DCN) do curso de graduação em nutrição (BRASIL, 2001; TREVISO e COSTA, 2017)

Neste contexto, caberá ao discente ter competência e desenvolver habilidade para realizar avaliação subjetiva global e que possibilite identificar a deficiência nutricional ou o estado nutricional, e assim dar sequência ao tratamento dietoterápico ou terapia nutricional (SOUSA JUNIOR et al., 2016).

Além de adquirir competência técnica, o discente deverá compreender, analisar e intervir os problemas inerentes a clínica, tendo como referência protocolos de avaliação, intervenção nutricional e monitoramento do estado clínico nutricional do paciente hospitalizado, a fim de viabilizar assistência nutricional para sistematizar, padronizar e aperfeiçoar os serviços na área de atuação (JUNQUEIRA e COTTA, 2014).

Em virtude da avaliação e diagnóstico nutricional serem habilidades adquiridas durante a formação acadêmica, conforme as DCN (2001) do curso de graduação em nutrição, justifica-se a realização da atual pesquisa, buscando verificar se os discentes estão aptos a realizar a ASG a partir de sua formação acadêmica, de acordo com suas competências e habilidades. 


\section{METODOLOGIA}

\section{Tipo de Estudo}

Trata-se de um estudo descritivo, analítico-exploratório e transversal; com abordagem quantitativa. A pesquisa quantitativa envolve a coleta sistemática de informação numérica, normalmente mediante condições de muito controle, além da análise dessa informação, utilizando procedimentos estatísticos (AYRES, 2007).

\section{População}

A população foi constituída de cem discentes, de ambos os sexos, regularmente matriculados no $8^{\circ}$ semestre do curso de graduação em nutrição de quatro Instituições de Ensino Superior (IES), de Belém (PA). Foram adotados os seguintes critérios de exclusão: discentes irregulares quanto a matrícula, os que estavam cursando os demais semestres de graduação e aqueles que não concordaram em assinar o Termo de Consentimento Livre e Esclarecido.

Por serem concluintes entende-se que os discentes devem apresentar aptidão para realizar a ASG, uma vez que é uma habilidade específica inerente à avaliação nutricional inserida no componente curricular.

\section{Aspectos Éticos}

O estudo foi aprovado pelo Comitê de Ética em Pesquisa com seres humanos do Conselho Nacional de Saúde, sob o número do CAAE: 78795617.1.0000.5169 e parecer número 2.363.116, garantindo os aspectos éticos pautados segundo os preceitos da Declaração de Helsinque e do Código de Nuremberg, assim como respeitando as normas de pesquisa envolvendo seres humanos do Conselho Nacional de Saúde (CNS), conforme Resolução CNS № 466/2012 (BRASIL, 2012). Enfatiza-se que as IES incluídas na pesquisa autorizaram a execução, mediante ofício e ciência do projeto, com destaque aos objetivos e metodologia. Destaca-se que a coordenação de cada IES informou aos discentes previamente sobre o escopo da pesquisa. 


\section{Protocolo de Coleta de Dados}

A ferramenta em questão é a ASG, caracterizada como um método simples, de custo muito baixo, com boa reprodutibilidade e confiabilidade. O desenvolvimento desse instrumento fundamenta-se em uma abordagem essencialmente clínica em forma de questionário, abordando a história clínica e exame físico do paciente (AZEVEDO et al., 2016).

A ASG valoriza a progressão de perda de peso do doente e o período em que ocorreu, além de outras variáveis clínicas significantes, como a existência de alterações do apetite, a presença de sintomas gastrointestinais e as mudanças da capacidade funcional (LIM, LIN e DANIELS, 2016; HANUSCH et al., 2016).

Outro tópico a ser analisado é o exame físico, que deverá ser realizado por meio do olhar clínico do examinador (observação, inspeção) e do tato (palpação), não incluindo equipamentos para análise destes parâmetros. A perda de tecido adiposo subcutâneo é melhor visualizada nas regiões do tríceps e bíceps, enquanto que a perda muscular no deltoide e quadríceps avalia o volume da massa muscular e tônus (AZEVEDO et al., 2016).

No final,combase na avaliação essencialmente subjetiva emrelação a cada um desses aspectos clínicos e físicos, o paciente é classificado em: A - bem nutrido; B - moderadamente desnutrido ou com suspeita de desnutrição, e C - gravemente desnutrido (LIM, LIN e DANIELS, 2016; SOUSA JUNIOR et. al., 2016).

Conforme o exposto, foi aplicado questionário semiestruturado, cujo roteiro foi composto pelas variáveis sexo e gênero e por sete perguntas fechadas inerentes à percepção dos discentes no processo de avaliação e diagnóstico nutricional pela ASG, verificando as habilidades dos discentes em sua execução, de acordo com os seguintes questionamentos: (1). Qual a finalidade de realizar a ASG? (2). Como se classifica o estado nutricional de acordo com a ASG? (3). Para fins de diagnóstico nutricional, quando a ASG deverá ser aplicada? (4). De que forma se avalia a ingestão alimentar? (5). Qual o período que os sintomas devem ser registrados pela ASG? (6). De que forma é analisada a capacidade funcional? (7). Quais os critérios para realizar o exame físico?

A coleta dos dados ocorreu no período de novembro de 2017 a janeiro de 2018, através de questionário autoaplicável, executado pelo autor da pesquisa em local reservado nas IES, 
durante o período de intervalo de aula e com tempo médio para as respostas de 20 minutos. O sigilo dos participantes foi garantido por meio da utilização do sistema alfanumérico, isto é, foram codificados pela letra $\mathrm{D}$ (discente), seguidos dos números dados aos questionários (D01), e as quatro Instituições de Ensino Superior pelas letras A, B, C, D.

\section{Análise dos Dados}

Os dados foram inferidos pela Análise de Correspondência Simples (AC), que objetiva verificar associações ou similaridades entre variáveis qualitativas ou variáveis contínuas categorizadas, apropriadas para dados nominais (CUNHA JUNIOR, 1997). Tem como fundamentação a representação ótima da estrutura de dados observados que geralmente são introduzidos sem qualquer tratamento estatístico prévio, provando a flexibilidade e utilidade da técnica. Sendo assim, a denominação do termo AC reflete estudar a correspondência entre as categorias das variáveis (RAMOS, ALMEIDA e ARAÚJO, 2008).

Portanto, a verificação de associação significativa entre as variáveis qualitativas pesquisadas foi realizada a partir das seguintes etapas: (1) Teste do Qui-quadrado, para verificar a dependência entre as variáveis, com nível de significância de 0,05; (2) Critério beta, valor maior que 3 indica que as variáveis dependentes apresentam risco $\leq 5 \%$; (3) Percentual de inércia, deverá ser maior que 70\%, logo as categorias das variáveis são aplicáveis e a associação é validada; e por fim, (4) Aplicação da análise de correspondência, por meio do software Statistica versão 8, para verificar as associações inferidas, que mostram o resíduo e o coeficiente de confiança das variáveis em análise no qual deve ser maior ou igual a 0,70 ou equivalentemente, $100 \times \gamma \%=70 \%$, observados a partir de tabelas.

\section{DISCUSSÃo}

A pesquisa foi composta por 100 discentes, distribuídos da seguinte forma e de acordo com a IES: A (n: 39); B (n: 23); C (n: 21) e D (n:17). A média de idade dos participantes foi de $25,49 \pm 7,12$ anos. Em relação ao total de alunos conforme o gênero verifica-se que $90 \%$ (n: 90 ) eram do sexo feminino e 10\% (n: 10) masculino. 
As variáveis estudadas foram inerentes aos dados referentes às habilidades e competências adquiridas no processo de diagnóstico nutricional a partir da ASG. Aplicou-se a Análise de Correspondência para verificar a associação das IES com as categorias das perguntas realizadas aos discentes.

A Tabela 1 apresenta resultado dos testes para verificar a dependência das variáveis em estudo ao nível de significância de 5\%, a partir do valor do qui-quadrado, p-valor e critério beta. Verifica-se então que as variáveis que tiveram condições viáveis para aplicação da técnica em estudo foram: IES x Finalidade da ASG, IES x Classificação da ASG e IES x Exame Físico ASG; isto é, a associação pela aplicação da técnica análise de correspondência correspondeu a percentuais superiores a $70 \%$.

Ressalta-se que as variáveis testadas e consideradas independentes não foram apresentadas, pois não houve associação.

Tabela 1 - Resultados do teste de dependência de variáveis: IES e dados referente as habilidades e competências sobre ASG. Belém. Ano 2018

\begin{tabular}{l|ccccccc}
\hline \multicolumn{1}{c|}{ VARIÁVEIS } & $\boldsymbol{\chi}^{2}$ & $\mathbf{g . 1 .}$ & $\mathbf{p}$ & $\mathbf{l}$ & $\mathbf{c}$ & $\boldsymbol{\beta}$ & Inércia \\
\hline IES x Finalidade da ASG & 27,91 & 15 & $<0,05$ & 4 & 6 & 3,33 & $>70 \%$ \\
IES x Classificação da ASG & 28,48 & 12 & $<0,05$ & 4 & 5 & 4,75 & $>70 \%$ \\
IES x Exame Físico ASG & 30,62 & 12 & $<0,05$ & 4 & 5 & 5,37 & $>70 \%$ \\
\hline
\end{tabular}

Nota: $\chi^{2}$ = qui-quadrado; g.l = grau de liberdade; $p$ = $p$-valor (nível de significância); 1 = número de linhas; $\mathrm{c}=$ número de colunas.

Fonte: Pesquisa de Campo.

Observa-se na tabela 2 os resíduos e grau de confiança das variáveis: IES e Finalidade da ASG. A pergunta realizada aos discentes foi: qual a finalidade de realizar a ASG? A resposta correta/esperada é a obtenção do diagnóstico nutricional do paciente.

Verifica-se que os discentes da IES-B e IES-D relataram que a finalidade da ASG é obter o diagnóstico nutricional e indicar a intervenção a ser realizada nos pacientes, com nível de significância de $87,89 \%$ e 71,75\%, respectivamente. Os discentes da IES-C disseram que a ASG 
é utilizada para fins de obter o diagnóstico nutricional, com associação estatística significativa entre as variáveis analisadas de $86,82 \%$.

Tabela 2 - Resíduos e Níveis de Confiança Resultantes da Aplicação da Análise de Correspondência às Variáveis: IES e Finalidade da ASG. Belém, Ano 2018

\begin{tabular}{c|cccccc}
\hline \multirow{2}{*}{ IES } & \multicolumn{5}{|c}{ FINALIDADE DA ASG } \\
\cline { 2 - 7 } & Diagnóstico & $\begin{array}{c}\text { Diagnóstico } \\
\text { Intervenção }\end{array}$ & $\begin{array}{c}\text { Risco } \\
\text { Nutricional }\end{array}$ & $\begin{array}{c}\text { Risco e } \\
\text { Diagnóstico }\end{array}$ & $\begin{array}{c}\text { Intervençã } \\
\text { o }\end{array}$ & Não \\
\hline \multirow{2}{*}{ A } & 0.95 & -0.20 & -0.22 & -0.62 & -0.62 & -0.62 \\
& 65.86 & 0.00 & 0.00 & 0.00 & 0.00 & 0.00 \\
B & -1.61 & 1.55 & -0.32 & -0.48 & -0.48 & -0.48 \\
& 0.00 & $\mathbf{( 8 7 . 8 9 )}$ & 0.00 & 0.00 & 0.00 & 0.00 \\
C & 1.51 & -1.83 & 1.01 & 1.02 & 0.90 & -0.62 \\
& $\mathbf{( 8 6 . 8 2 )}$ & 0.00 & 68.75 & 69.23 & 63.19 & 0.00 \\
D & -1.24 & 1.07 & -1.01 & -0.41 & -0.41 & -0.41 \\
& 0.00 & $\mathbf{( 7 1 . 7 5 )}$ & 0.00 & 0.00 & 0.00 & 0.00 \\
\hline
\end{tabular}

Fonte: Pesquisa de Campo.

De acordo com os resultados da atual pesquisa, a ASG foi associada com o diagnóstico nutricional e intervenção a ser realizada nos pacientes em duas instituições de ensino. Segundo Azevedo et al. (2016), sabe-se que ASG não indica a terapia nutricional a ser realizada, e sim, a partir do diagnóstico da mesma, logo dará respaldo ao nutricionista a executar o planejamento dietoterápico mais adequado.

É parte integrante da estratégia de ensino da avaliação nutricional no curso de graduação em nutrição gerar no estudante o correto entendimento da finalidade das ferramentas e indicadores nutricionais, além de identificar os fatores de risco nutricional, realizar a avaliação nutricional do paciente e fornecer o diagnóstico nutricional (TREVISO e COSTA, 2017). 
Dessa forma, as competências e habilidades exercem uma grande importância no processo de ensino e de aprendizagem, permitindo a capacidade de contextualizar demandas complexas de um determinado tema e assunto, além de integrar saberes essenciais na forma de planejar e executar os conhecimentos adquiridos (COSTA et al., 2013)

Para isso, um bom planejamento e adequada construção dos Projetos Pedagógicos dos Cursos de Nutrição visa potencializar a articulação entre os conteúdos das diferentes disciplinas e integrar melhor os aspectos teóricos e práticos do currículo. Logo, permitirá que o assunto abordado nas disciplinas seja visto pelo discente de forma significativa e desafiadora, baseado em estratégias de problematizar a realidade concreta e aumentar o tempo de vivência e espaços dos discentes nas IES onde a prática se realiza (JUNQUEIRA e COTTA, 2014).

Sendo assim, o profissional deverá estar capacitado para reconhecer estruturas e processos orgânicos patológicos e suas inter-relações com a alimentação e nutrição; interpretar as diversas situações da área da nutrição clínica, entre elas a avaliação e diagnóstico nutricional de pacientes, a fim de estar preparado para o mercado de trabalho, bem como buscar a integração com as equipes multidisciplinares, valorização, ética, humanização e a promoção da alimentação saudável, na intervenção para manutenção ou recuperação do estado nutricional do paciente (COSTA et al., 2013).

A Tabela 3 destaca os resíduos e grau de confiança das variáveis: IES e Classificação da ASG. Quanto à classificação da ASG, os discentes foram questionados a respeito do diagnóstico nutricional do paciente quando fosse classificado com a letra C. A resposta corresponde a “Gravemente Desnutrido".

Observa-se que os discentes da IES-A responderam que não lembram o diagnóstico da ASG de acordo com a letra C, apresentando uma associação forte estatisticamente (98,61\%). Enquanto os alunos da IES-B relataram que o diagnóstico significa bem nutrido com nível de significância de 73,98\%; e por fim, os discentes da IES-C informaram que o diagnóstico é gravemente desnutrido com associação estatística significativa entre as variáveis analisadas de $73,18 \%$. 
Tabela 3 - Resíduos e Níveis de Confiança Resultantes da Aplicação da Análise de Correspondência às Variáveis: IES e Classificação da ASG. Belém, Ano 2018

\begin{tabular}{|c|c|c|c|c|c|}
\hline \multirow[b]{2}{*}{ IES } & \multicolumn{5}{|c|}{ CLASSIFICAÇÃO DA ASG } \\
\hline & $\begin{array}{c}\text { Bem } \\
\text { Nutrido }\end{array}$ & Levemente & Moderadamente & Gravemente & $\begin{array}{c}\text { Não } \\
\text { lembro }\end{array}$ \\
\hline \multirow{2}{*}{$\mathrm{A}$} & -0.45 & 0.43 & -0.20 & 0.83 & 2.46 \\
\hline & 0.00 & 33.39 & 0.00 & 59.13 & (98.61) \\
\hline \multirow{2}{*}{ B } & 1.13 & -0.32 & -1.48 & -1.80 & 0.75 \\
\hline & (73.98) & 0.00 & 0.00 & 0.00 & 54.67 \\
\hline \multirow{2}{*}{$\mathrm{C}$} & -0.92 & -0.23 & 0.27 & 1.11 & -2.47 \\
\hline & 0.00 & 0.00 & 21.28 & (73.18) & 0.00 \\
\hline \multirow{2}{*}{$\mathrm{D}$} & 0.39 & -0.02 & -0.49 & -0.38 & 0.61 \\
\hline & 30.20 & 0.00 & 0.00 & 0.00 & 45.54 \\
\hline
\end{tabular}

Fonte: Pesquisa de Campo.

Os resultados significativos mostram equívoco no diagnóstico nutricional dado pelos discentes de duas IES. Logo, vários fatores podem estar contribuindo para esse resultado na relação discentes $\mathrm{x}$ docente $\mathrm{x}$ instituição de ensino, entre eles: a ausência ou poucas aulas práticas em torno da ASG executadas; inexperiência prática de preceptores e docentes; inexistência de atividades integradas das disciplinas da área clínica; estratégias metodológicas ativas não contempladas nos projetos pedagógicos; atividades práticas apenas no fim do curso, diminuindo o contato com a realidade e domínio das habilidades, entre outros.

As aulas práticas são importantes para o entendimento do conhecimento adquiridos nas aulas teóricas, já que a vivência de uma experiência facilita a fixação das informações. A prática inclui atividades em campo a nível hospitalar, permitindo aprendizagem a partir da vivência, conhecimento amplo na área clínica e desenvolvimento de capacidade de trabalho em equipe. Isto promove nos alunos reflexão, construção de ideias e atitudes, além da competência de procedimentos e habilidades adquiridas (BASSOLI, 2014). 
Considera-se que o diagnóstico nutricional errado refletirá em conduta nutricional inadequada, piorando assim, o estado clínico e nutricional do paciente. Portanto, a precisão diagnóstica da ASG depende das habilidades do observador, visto ser um método subjetivo e requer treinamento prático adequado (DIAS, 2011).

Neste contexto, as habilidades e competências devem ser definidas com referência a ASG e os discentes deverão ser capazes de compreender e dominar a técnica de avaliação. Para isso, caberá ao docente e IES assimilarem as dificuldades inerentes ao diagnóstico nutricional, buscando associar as noções saber, saber-fazer e direcionar as atividades (ou tarefas) em que eles poderão se materializar e se fazer compreender (JUNQUEIRA e COTTA, 2014).

A exposição do estudante ao ensino de avaliação nutricional desde o primeiro semestre do curso e de forma contínua não é vista nos Projetos Pedagógicos das Instituições. o envolvimento do discente com a prática clínica desde o início de sua formação acadêmica visa à aprendizagem baseada em conhecimentos teóricos de uma das áreas mais importantes da nutrição. Mesmo em seu primeiro ano, os discentes são capazes de construir conhecimentos e habilidades a respeito da avaliação e diagnóstico nutricional, logo, enriquece sua compreensão a respeito dos processos saúde versus doença (BASSOLI, 2014).

A definição dos focos da aprendizagem, embora essencial para o sucesso institucional, além de controversa, não tem valia se a forma como se pretende alcançar esses objetivos não aborde o treinamento prático, protocolos previamente definidos e testados pelos discentes, e ainda, encontrar receptividade no aluno para executar a avaliação e saber reconhecer e interpretar as anormalidades observadas, visto que é um método essencialmente subjetivo (JUNQUEIRA e COTTA, 2014).

Nesse processo, o professor terá que ter a consciência da responsabilidade pela formação de futuros profissionais (nutricionistas clínicos) e a busca na construção de estratégias metodológicas ativas com o objetivo de continuar aprendendo e melhorando a abordagem didático-pedagógica utilizada. Enfatiza-se que as IES deverão valorizar o docente, capacitando-o para que o processo de formação continuada seja aperfeiçoado. Além disso, o docente deverá enfocar o ensino reflexivo, humanístico e crítico, a fim de desafiar, instigar, 
contribuir, humanizar e estimular os discentes na construção de habilidades e competências que fortaleçam o compromisso profissional (BASSOLI, 2014; TREVISO e COSTA, 2017).

A Tabela 4 mostra os resíduos e grau de confiança das variáveis: IES e Exame Físico segundo a ASG. A pergunta realizada aos discentes foi: o exame físico a partir da ASG deverá ser realizado de que forma? A resposta corresponde: O exame físico deverá ser realizado por meio do olhar clínico do examinador (observação, inspeção) e do tato (palpação), não incluindo equipamentos para análise destes parâmetros.

Nota-se que houve associação entre a palpação e a IES-A, com significância estatística de 75,11\%. Em relação aos discentes da IES-B houve associação forte com palpação, inspeção e instrumento com nível de significância de 97,87\%. Os discentes da IES-C apresentaram associação significativa forte com a inspeção, com 99,68\% de significância.

Tabela 4 - Resíduos e Níveis de Confiança Resultantes da Aplicação da Análise de Correspondência às Variáveis: IES e Exame Físico segundo da ASG. Belém, Ano 2018

\begin{tabular}{|c|c|c|c|c|c|}
\hline \multirow{2}{*}{ IES } & \multicolumn{5}{|c|}{ EXAME FÍSICO ASG } \\
\hline & Inspeção & Palpação & $\begin{array}{l}\text { Palpação e } \\
\text { Inspeção }\end{array}$ & $\begin{array}{l}\text { Palpação, Inspeção e } \\
\text { Instrumento }\end{array}$ & $\begin{array}{l}\text { Não } \\
\text { lembro }\end{array}$ \\
\hline \multirow{2}{*}{ A } & -0.20 & 1.15 & 0.43 & -0.71 & 0.98 \\
\hline & 0.00 & (75.11) & 33.30 & 0.00 & 67.13 \\
\hline \multirow{2}{*}{ B } & -1.24 & -0.96 & -1.61 & 2.30 & -0.48 \\
\hline & 0.00 & 0.00 & 0.00 & (97.87) & 0.00 \\
\hline \multirow{2}{*}{ C } & 2.95 & 0.17 & 0.49 & -1.96 & -0.46 \\
\hline & (99.68) & 13.86 & 37.85 & 0.00 & 0.00 \\
\hline \multirow{2}{*}{$\mathrm{D}$} & -1.54 & -0.82 & 0.67 & 0.58 & -0.41 \\
\hline & 0.00 & 0.00 & 49.64 & 43.72 & 0.00 \\
\hline
\end{tabular}

Fonte: Pesquisa de Campo. 
Destaca-se que os discentes de todas as instituições pesquisadas enfatizaram a inspeção ou a palpação de forma isolada, bem como o uso de instrumentos. De acordo com Souza et al. (2018), o exame físico trata-se de um procedimento de simples execução, a partir do processo de inspeção e palpação associados, permitindo análise de depleções de massa muscular e tecido adiposo subcutâneo no paciente, além do volume da massa e tônus do músculo. Assim, o exame físico é fundamental no processo de diagnóstico nutricional, contribuindo na detecção da desnutrição que corresponde a $20 \%$ a $78,9 \%$ nos pacientes hospitalizados.

Entre as fragilidades observadas na realização do exame físico em dar o diagnóstico nutricional, destaca-se a dificuldade na construção de habilidades em detectar anormalidades nutricionais inerentes aos pacientes, por falta de treinamento prático adequado ou por conhecimento adquirido de forma equivocada. Esta realidade poderá trazer consequências graves no processo de intervenção nutricional do paciente. O diagnóstico nutricional errado do paciente condiz em uma conduta nutricional inadequada pelo nutricionista (HANUSCH et al., 2016).

Segundo as DCN (2001) do Curso de Graduação em Nutrição, o nutricionista tem que ter competência e habilidades específicas como: avaliar, diagnosticar e acompanhar o estado nutricional; identificar as alterações físicas e funcionais; e valorizar a progressão de perda de peso em relação ao tempo de doença (AZEVEDO et al., 2016; TREVISO e COSTA, 2017).

Dessa forma, entende-se que as IES deverão contemplar na matriz curricular as competências e habilidades específicas a fim de formar profissionais capacitados nas diversas áreas de atuação, entre elas, a nutrição clínica, a fim de nortear o acadêmico durante o processo de ensino e de aprendizagem (JUNQUEIRA e COTTA, 2014).

A competência não está alinhada apenas com o conhecimento técnico, como realizar anamnese nutricional e exame físico, mas também com capacidade de reflexão, processo humanizado, integralidade, diálogo e atualização, e por fim conhecimentos, habilidades e atitudes necessárias para que os discentes ajam de maneira ativa, responsável e criativa na área da nutrição clínica (COSTA, et al., 2013; JUNQUEIRA e COTTA, 2014).

A abordagem utilizada para facilitar a aprendizagem dos discentes é a busca de um cenário de rápidas transformações, estas estratégias de ensino necessitam ser remodeladas 
para que seu principal objetivo, formar bons profissionais, seja alcançado. Nessa perspectiva alguns pontos são propostos, como antecipar as atividades práticas e obter o contato mais precoce com a realidade, isto é, com o meio hospitalar; criar práticas integradas na área clínica e social; correlacionar conteúdos entre as disciplinas da área clínica, que devem ser organizadas em processo crescente de complexidade; ampliar quadro de disciplinas optativas; e organizar o curso, de modo a permitir ao discente desenvolver outras atividades teóricas e práticas extracurriculares (TREVISO; COSTA, 2017).

A síntese dos resultados desta pesquisa permitiu construir um esquema representacional (figura 1), considerando que o diagnóstico nutricional obtido pela ASG é fundamentado na transmissão de conhecimentos, no desenvolvimento de competências, habilidades e atitudes; isto é, mediante a relação teoria e prática das disciplinas na área de nutrição clínica. A relação está pautada no saber-conhecer (conhecimentos teóricos de âmbito profissional ou acadêmico na área clínica); saber-fazer (conjunto de destrezas e habilidades cognitivas para aplicar a ASG de acordo com o conhecimento que possui); saberser (ajustes de valores, princípios, ética, moral e atitudes profissionais válidas nas áreas de atuação); saber-conviver (atitudes pessoais e interpessoais, que facilite a convivência e o trabalho com os demais profissionais na área hospitalar, em equipe) e saber utilizar (aperfeiçoar-se estrategicamente o conhecimento a partir das competências) (TREVISO; COSTA, 2017). 
Figura 1. Esquema representacional referente a relação teoria e prática na área de nutrição clínica

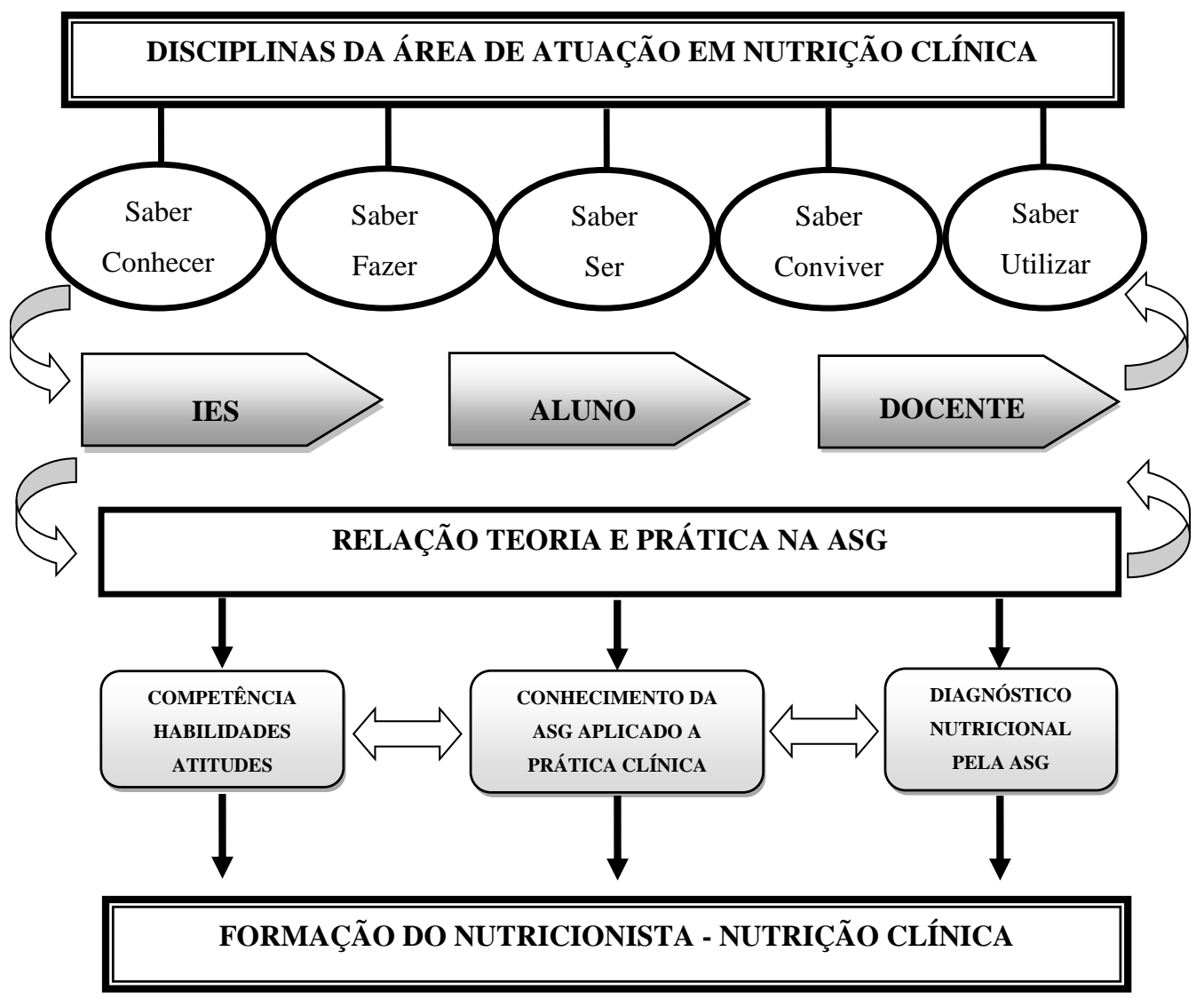

Fonte: Esquema elaborado pelo autor da pesquisa, 2018.

A relação entre a teoria e a prática no processo da avaliação nutricional buscando o diagnóstico do paciente pela ASG, assegura o discente a desenvolver suas habilidades, articular experiências e obter a competência clínica durante sua formação acadêmica, principalmente na utilização de ferramentas de diagnóstico nutricional como a ASG (HANUSCH et al., 2016).

No contexto da pesquisa, o saber-conhecer está relacionado com a compreensão e interpretação das questões abordadas na ASG; o saber-fazer, em garantir aos discentes as habilidades cognitivas para aplicar a ASG a partir da realização da prática de forma contínua a nível hospitalar; o saber-ser propõe ao discente ser um profissional ético e moral, mantendo uma relação discente $\mathrm{x}$ paciente $\mathrm{x}$ docente pautada nos valores, princípios e atitudes profissionais válidas nas áreas de atuação de forma ética e moral; o saber-conviver, estabelecer uma abordagem clara e compreensiva da ferramenta que será aplicada ao 
paciente, que o mesmo tire todas as dúvidas a respeito do procedimento, mantendo sempre os direitos e o respeito aos mesmos; e por fim, o saber utilizar, que corresponde ao aperfeiçoamento das competências a partir dos conhecimentos adquiridos (TREVISO; COSTA, 2017).

Os saberes devem integrar a construção do processo de ensino e de aprendizagem pactuadas com as IES - Discente - Docente, promoverão a inserção do discente na sua realidade profissional, a partir de uma ação transformadora como centro do processo de ensinar e apreender.

Assim, as ações inerentes a nutrição clínica serão articuladas de acordo com o conhecimento teórico e prático, aplicando as habilidades conforme suas próprias reflexões e vivências acadêmicas.

\section{CONCLUSÃO}

De acordo com a análise por correspondência, observam-se associações entre as IES versus finalidade, classificação e exame físico. A ASG foi associada com o diagnóstico nutricional e intervenção a ser realizada nos pacientes, os discentes de todas as instituições pesquisadas enfatizaram a inspeção ou a palpação de forma isolada, bem como o uso de instrumentos, além de equívoco no diagnóstico nutricional.

Destaca-se dificuldade em realizar técnicas de avaliação nutricional como ferramenta de diagnóstico por quantidade insuficiente de aulas práticas, tornando uma grande limitação nas habilidades e competências adquiridas no âmbito acadêmico.

Há necessidade de estratégias que fomentem a necessidade de realizar ajustes, construções e reformulações que deverão ser compreendidas e avaliadas de forma contínua de acordo com a vivência dos discentes e nos resultados por eles alcançados enquanto protagonistas do processo de ensino e de aprendizagem permitindo que o discente possa experimentar novas experiências positivas no desenvolvimento de competências, habilidades, conhecimentos e atitudes, atrelado ao bom desempenho do papel facilitador do docente. 
A partir desse estudo, surgem novas possibilidades de continuidade da pesquisa que tenham foco as estratégias implementadas nas IES, de modo a ampliar o processo de ensino e aprendizagem na área da nutrição clínica.

\section{REFERÊNCIAS}

ANDRADE, Patrícia Amaro; SANTOS, Carolina Araújo dos; FIRMINO, Heloísa Helena; ROSA, Carla de Oliveira Barbosa. Importância do rastreamento de disfagia e da avaliação nutricional em pacientes hospitalizados. Einstein, v.16,n.2, p. 01-06, 2018. Disponível em: <http://www.scielo.br/pdf/eins/v16n2/pt_1679-4508-eins-16-02-eA04189.pdf 5 .Acesso em: 11 out. 2018.

AQUINO, Rita de Cássia Aquino; PHILIPPI, Sônia Tucunduva. Desenvolvimento e avaliação de instrumentosdetriagem nutricional. Rev Bras Enferm, Brasília, v. 65, n. 4, p. 607-13. 2012. Disponível em: <https://core.ac.uk/download/pdf/37514970.pdf >. Acesso em: 22 mai. 2016.

AYRES, Manuel; AYRES JUNIOR, Manuel; AYRES, Daniel; SANTOS, Regis Sivori Silva dos. BioEstat 5.0: aplicações estatísti cas nas áreas das ciências biológicas e médicas. Belém: MCT; IDSM; CNPq, p. 364. 2007.

AZEVEDO, Arúquia Souza; OLIVEIRA, Dayane Cardoso; SOARES, Priscilla Kálisy. Perfil Nutricional de PacientesAdultos e Idosos Hospitalizados. Revista Saúde e Pesquisa, v. 9, n. 1, p. 25-29. 2016. Disponível em: <http://periodicos.unicesumar.edu.br/index.php/saudpesq/article/view/4706/2746>. Acesso em: 15 jan. 2017.

BASSOLI, Fernanda. Atividades práticas e o ensino-aprendizagem de ciência (s): mitos, tendências e distorções.Ciênc. Educ, v. 20, n. 3, p. 579-593, 2014. Disponível em: <http://www.scielo.br/pdf/ciedu/v20n3/1516-7313-ciedu-20-030579.pdf>. Acesso em: 24 abr. 2016.

BRASIL. Conselho Nacional de Saúde. Resolução 466, de 12 de dezembro de 2012. Aprova as diretri zes e normas regulamentadoras de pesqui sa envolvendo seres humanos. Diário Oficial da União, Brasília, n. 12, 13 de junho de 2013 - seção 1, página 59. Disponível em: <http://conselho.saude.gov.br/resolucoes/2012/reso466.pdf>.Acessoem:09 set. 2015.

BRASIL. Ministério da Educação. Conselho Nacional de Educação. Câmara de Educação Superior. ResoluçãoCNE/CES no5, de 7 de novembro de 2001. Insti tui di retri zes curri culares naci onais do curso de graduação em nutrição. Diário Oficial da União. Brasília, 2011.39 p. Disponível em: <http://portal.mec.gov.br/cne/arquivos/pdf/CES05.pdfs. Acesso em: 23 out. 2012.

COSTA, Ester de Queiroz; DOMINGUES, Josiane Roberto; MALHEIROS, Luciana Reis; JARDIM, Maria de Fátima Barros. Desafios à reforma curricular em um curso de graduação em nutrição. Demetra, v. 8, n. 3, p. 469-4 85, 2013.Disponível em: <https://www.e-publicacoes.uerj.br/index.php/demetra/article/viewFile/6210/7108>. Acesso em: 19 fev. 2015.

CUNHA JUNIOR, Marcus Vinicius Moretti da. Análise Multidimensional de Dados Categóricos: A aplicação das análises de correspondência simples e múltipla em marketing e sua integração com técnicas de análise de dados quantitativos. Rio Grande do Sul: UFRGS, 1997.

DETSKY AS; MCLAUGHLIN JR; BAKER JP; JOHNSTON N; WHITTAKER S; MENDELSON RA; JEEJEEBHOY KN. What is subjective global assessment of nutritional status? JPEN J Parenter Enteral Nutr, v.11, p. 8-13. 1987. Disponível em: <https://www.ncbi.nlm.nih.gov/pubmed/3820522>. Acesso em: 22 mai. 2013.

DIAS MCG; VAN AANHOLT DPJ; CATALANI LA; REY JSF; GONZALES MC; COPPINI L; FRANCO FILHO JW; PAES-BARBOSA MR; HORIE L; ABRAHÃO V; MARTINS C. Projeto Di retri zes - Triagem e Avaliação do Esta do Nutricional.Associação Médica Brasileira e Conselho Federal de Medicina, Volume IX, 2011. Disponível em: <https://diretrizes.amb.org.br/_BibliotecaAntiga/triagem_e_avaliacao_do_estado_nutricional.pdf>.Acessoem:07 ago. 2012. 
DOMINGUES LCB; NEMETZ KB; CRUZ LB; GREGIANIN LJ. Avaliação Nutricional Subjetiva Global: aplicabilidade em crianças e adolescentes com neoplasia maligna - Resultados Preliminares. Clin Biomed Res. v. 38, n. 3, 2018. Disponível em: <https://seer.ufrgs.br/hcpa/article/view/80224/pdf>. Acesso em: 12 nov. 2018.

HANUSCH, Flávia Daysa; SILVA, Maria da Guia Bezerra da; PRADO, Leila Virgínia da Silva; COSTA, Milena Damasceno de Souza; GADELHA, Patrícia Calado Ferreira Pinheiro. Avaliação nutricional de pacientes submetidos à cirurgia do trato gastrointestinal: associação entre avaliação subjetiva global, ferramentas de triagem nutricional e métodos objet ivos. Nutr. Clín. Diet. Hosp., v. 36, n. 2, p. 10-19, 2016. Disponível em: <http://revista.nutricion.org/PDF/daysa.pdf>. Acesso em: 10 jun. 2017.

JUNQUEIRA, Túlio da Silva; COTTA, Rosângela Minardi Mitre. Matriz de ações de alimentação e nutrição na Atenção Básica de Saúde: referencial para a formação do nutricionista no contexto da educação por competências. Ciênc. Saúde Coletiva. v. $19, \quad$ n. $5, \quad$ p. $1459-1474, \quad 2014 . \quad$ Disponível <https://www.scielosp.org/article/ssm/content/raw/?resource_ssm_path=/media/assets/csc/v19n5/1413-8123-csc-1905-01459.pdf>. Acesso em: 13 set. 2015.

LIM, S. L.; LIN, X. H.; DANIELS, L. Seven-Point Subjective Global Assessment Is More Time Sensitive Than Conventional Subjective Global Assessment in Detecting Nutrition Changes. JPEN J Parenter Enteral Nutr. v. 40, n. 7, p. 966-972, 2016. Disponível em: <https://www.ncbi.nlm.nih.gov/pubmed/25847315>. Acesso em: 27 nov. 2018.

MALONE, A.; HAMILTON, C. The Academy of Nutrition and Dietetics/The American Society for Parenteral and Enteral Nutrition Consensus malnutrition characteristics: application in practice. Nutr Clin Pract. v. 28, n. 6, p. 639-650, 2013. Disponível em: <https://www.ncbi.nlm.nih.gov/pubmed/24177285>. Acesso em: 30 jul. 2015.

PAZ, Laryssa de Souza Cirqueira; COUTO, Amanda do Vale. Avaliação nutricional em pacientes críticos: revisão de literatura. BRASPEN J., v. 31, n. 3, p. 269-277, 2016. Disponível em: <http://www.braspen.com.br/home/wpcontent/uploads/2016/11/16-Avalia\%C3\%A7\%C3\%A3o-nutri-em-pacentes-criticos.pdf>. Acesso em: 01 nov. 2017.

RAMOS, Edson Marcos Leal Soares; ALMEIDA, Silva dos Santos; ARAÚJO, Adrilayne, dos Reis. Segurança pública: Uma abordagem estatística e computacional, v. 2, Belém: EDUFPA, 2008.

RASLAN M, GONZALEZ MC, DIAS MC, NASCIMENTO M, CASTRO M, MARQUES P, SEGATTO S, TORRINHAS RS, CECCONELLO I, WAITZBERG DL. Comparison of nutritional risk screening tools for predicting clinical outcomes in hospitalized patients. Nutr, v. 26, n. 7-8, p.721-26, 2010. Disponível em: <https://www.ncbi.nlm.nih.gov/pubmed/19963352/>. Acesso em: 03 jan. 2012.

SANTOS, Alexsandro Ferreira dos; RABELO JUNIOR, Antônio Aragão; CAMPOS, Fernanda Larissa Brito; SOUSA, Rosângela Maria Lopes de; VELOSO, Helma Jane Ferreira; CHEIN, Maria Bethânia da Costa. Scored patient-generated Subjective Global Assessment: Length of hospital stay and mortality in cancer patients. Rev. Nutri. Campinas, v. 30, n. 5, p. 545-553, 2017. Disponível em: <http://www.scielo.br/scielo.php?script=sci arttext\&pid=S1415-52732017000500545>. Acesso em: 22 mar. 2018.

SOUSA JUNIOR, Josimar Barbosa; CASTRO, Teila; LIMA, Laís; BATISTA, Fábio. Comparação entre avaliação subjetiva global e o novo diagnóstico nutricional proposto pela ASPEN em pacientes cirúrgicos. BRASPEN J, v. 31, n. 4, p. 305-310, 2016. Disponível em: <http://www.braspen.com.br/home/wp-content/uploads/2017/02/05-AO-Compra\%C3\%A7\%C3\%A30entre-avalia\%C3\%A7\%C3\%A3o-subjetiva.pdf>. Acesso em: 16 set. 2018.

SOUZA, Guilherme Silva Freire de; SILVA, Elisiane Beatriz da; CORDEIRO, Susana Arruda; OLIVEIRA, Natália Dantas de; MOURA, Renally de Lima; DANTAS, Emelly Naiara dos Anjos; MACEDO, Nahayanne Louise da Silva; MACIEL, Fernanda Fátima Costa; OLIVEIRA, Davi Aires de; SILVA, Jordania Candice Costa; DONATO, Nilcimelly Rodrigues. Contribuição da semiologia para o diagnóstico nutricional de pacientes hospitalizados. International Journal of Nutrology, v. 11, n.1, 2018. Disponível em: <https://www.thieme-connect.com/products/ejournals/abstract/10.1055/s-0038-1670721>. Acesso em: 15 dez. 2018.

TREVISO, Patrícia.; COSTA, Bartira Ercília Pinheiro. Percepção de profissionais da área da saúde sobre a formação emsua atividade docente. Texto Contexto Enferm, v. 26, n. 1, p. 01-09, 2017. Disponível em: <http://www.scielo.br/pdf/tce/v26n1/pt 0104-0707-tce-26-01-e5020015.pdf>. Acesso em: 21 jun. 2018.

\section{$(\mathrm{cc}) \mathrm{EY}$}

Este trabalho está licenciado com uma Licença Creative Commons - Atribuição 4.0 Internacional. 